\title{
O Impacto da COVID-19 no Diagnóstico de Doenças Cardíacas na América Latina Uma Subanálise do INCAPS COVID
}

\section{The Impact of COVID-19 on Diagnosis of Heart Disease in Latin America an INCAPS COVID Sub-analysis}

Rodrigo Julio Cerci, ${ }^{\circledR}$ João Vicente Vitola, ${ }^{1}$ Diana Paez, ${ }^{2}$ Alejandro Zuluaga, ${ }^{3 \oplus}$ Marcio Sommer Bittencourt, ${ }^{4 \oplus}$ Lilia M. Sierra-Galan, ${ }^{5}$ Patricia Carrascosa, ${ }^{6}$ Roxana Campisi, ${ }^{6,7}{ }^{6}$ Claudia Gutierrez-Villamil, ${ }^{8}$ Amalia Peix, ${ }^{9}$ Duane Chambers, ${ }^{10}$ Mayra Sánches Velez, ${ }^{11}$ Carla M. G. Alvarado, ${ }^{12}$ Ana C. F. Ventura, ${ }^{13}$ Alejandro Maldonado, ${ }^{14}$ Alfredo P. Castanos, ${ }^{15}$ Teresa C. Diaz, ${ }^{16}$ Yariela Herrera, ${ }^{17}$ Manuel C. Vasquez, ${ }^{18}$ Ana A. Arrieta, ${ }^{19}$ Fernando Mut, ${ }^{20}$ Cole Hirschfeld, ${ }^{21}$ Eli Malkovskiy, ${ }^{21,22}$ Benjamin Goebel, ${ }^{23}{ }^{12}$ Yosef Cohen, ${ }^{24}$ Michael Randazzo, ${ }^{21}$ Leslee J. Shaw, ${ }^{23}$ Michelle C. Williams, ${ }^{25}$ Todd C. Villines, ${ }^{26}$ Nathan Better, ${ }^{27}$ Sharmila Dorbala, ${ }^{28}$ Paolo Raggi, ${ }^{29}$ Thomas N. B. Pascual, ${ }^{30}$ Yaroslav Pynda, ${ }^{31}$ Maurizio Dondi, ${ }^{31}$ Andrew J. Einstein ${ }^{21,22}$

Quanta Diagnóstico por Imagem - Cardiovascular CT, ${ }^{1}$ Curitiba, PR - Brasil

International Atomic Energy Agency - Division of Human Health, ${ }^{2}$ Vienna - Áustria

Cedimed Quirosalud, Universidad Pontificia Bolivariana y Universidad CES, ${ }^{3}$ Medelin - Colômbia

Diagnósticos da América SA - DASA, ${ }^{4}$ Barueri, SP - Brasil

American British Cowdray Medical Center IAP,5 Mexico City, Mexico City - México

Diagnostico Maipu, ${ }^{6}$ Buenos Aires - Argentina

Instituto Argentino de Diagnostico y Tratamiento S.A, ${ }^{7}$ Buenos Aires - Argentina

Fundación Cardioinfantil-Instituto de Cardiologia, ${ }^{8}$ Bogota - Colômbia

Institute of Cardiology and Cardiovascular Surgery - Department of Nuclear Medicine, ${ }^{9}$ La Habana - Cuba

Radiology West, ${ }^{10}$ Montego Bay - Jamaica

Carlos Andrade Marin Specialty Hospital, ${ }^{11}$ Quito - Equador

Tecnodiagnosis, ${ }^{12}$ Guatemala - Guatemala

Radiology Clinic Brito Mejia Pena, ${ }^{13}$ San Salvador - El Salvador

Servicio de Medicina Nuclear, ${ }^{14}$ Tegucigalpa - Honduras

Centro Diagnostico Especializado, ${ }^{15}$ Santo Domingo - República Dominicana

Hospital Salud Integral, ${ }^{16}$ Panama City - Panamá

Hospital Santo Tomas - Nuclear Medicine Section, ${ }^{17}$ Panama - Panamá

Hospital Central, Instituto de Prevision Social, ${ }^{18}$ Asuncion - Paraguai

Hospital Dr. Rafael Angel Calderon Guardia, ${ }^{19}$ San Jose - Costa Rica

Italian Hospital - Nuclear Medicine Service, ${ }^{20}$ Montevideo - Uruguai

Columbia University Irving Medical Center and New York-Presbyterian Hospital - Department of Medicine, ${ }^{21}$ New York - Estados Unidos da América Seymour, Paul and Gloria Milstein Division of Cardiology, ${ }^{22}$ New York - Estados Unidos da América

Weill Cornell Medical College and New York-Presbyterian Hospital, ${ }^{23}$ New York - Estados Unidos da América

Technion Israel Institute of Technology, ${ }^{24}$ Haifa - Israel

BHF Centre for Cardiovascular Science, University of Edinburgh, ${ }^{25}$ Edinburg - Reino Unido da Grã-Bretanha

University of Virginia, ${ }^{26}$ Charlottesville, Virginia - Estados Unidos da América

Royal Melbourne Hospital and University of Melbourne, ${ }^{27}$ Melbourne - Austrália

Brigham and Women's Hospital, ${ }^{28}$ Boston, Massachusetts - Estados Unidos da América

University of Alberta - Department of Medicine and Division of Cardiology, ${ }^{29}$ Edmonton, Alberta - Canadá

Philipines Nuclear Research Institute, ${ }^{30}$ Manila - Filipinas

International Atomic Energy Agency - Division of Human Health, ${ }^{31}$ Vienna - Áustria

Correspondência: Rodrigo Julio Cerci •

Quanta Diagnóstico por Imagem - Cardiovascular CT - Almirante Tamandaré, 1000. CEP 80035-170, Curitiba, PR - Brasil

E-mail: rjcerci@gmail.com

Artigo recebido em 04/05/2021, revisado em 09/06/2021, aceito 09/06/2021

DOI: https://doi.org/10.36660/abc.20210388 


\title{
Resumo
}

Fundamento: A pandemia de COVID-19 interferiu na prestação de atendimento a doenças cardiovasculares na América Latina. No entanto, o efeito da pandemia nos volumes de procedimentos cardíacos diagnósticos ainda não foi quantificado.

Objetivo: Avaliar (1) o impacto de COVID-19 nos volumes de diagnóstico cardíaco na América Latina e (2) determinar sua relação com a incidência de casos de COVID-19 e as medidas de distanciamento social.

Métodos: A International Atomic Energy Agency realizou uma pesquisa mundial avaliando mudanças nos volumes diagnósticos cardíacos decorrentes da COVID-19. Foram obtidos os volumes diagnósticos cardíacos dos locais participantes para março e abril de 2020 e comparados com março de 2019. Foram coletados dados de distanciamento social a partir dos Relatórios de mobilidade da comunidade de Google e a incidência de COVID-19 por país a partir de Our World in Data.

Resultados: Foram realizadas pesquisas em 194 centros que realizam procedimentos diagnósticos cardíacos, em 19 países da América Latina. Em comparação com o mês de março de 2019, os volumes dos procedimentos diagnósticos cardíacos diminuíram 36\% em março de 2020 e 82\% em abril de 2020.As maiores reduções ocorreram em relação aos testes de estresse ecocardiográfico $(91 \%)$, testes ergométricos de esteira $(88 \%)$ e escore de cálcio por tomografia computadorizada (87\%), com pequenas variações entre as sub-regiões da América Latina. As mudanças em padrões de distanciamento social $(p<0,001)$ estavam mais fortemente associadas com a redução do volume do que a incidência de COVID-19 $(p=0,003)$.

Conclusões: A COVID-19 foi associada a uma redução significativa de procedimentos diagnósticos cardíacos na América Latina, a qual foi mais relacionada ao distanciamento social do que ao aumento da incidência da COVID-19. São necessários melhor equilíbrio e timing de medidas de distanciamento social e planejamento para manter o acesso ao atendimento médico durante um surto pandêmico, especialmente em regióes com alta mortalidade cardiovascular.

Palavras-chave: Teste Cardíaco; Coronavírus; COVID-19; Doença Cardiovascular; Saúde global.

\begin{abstract}
Background: The COVID-19 pandemic has disrupted the delivery of care for cardiovascular diseases in Latin America. However, the effect of the pandemic on the cardiac diagnostic procedure volumes has not been quantified.

Objective: To assess (1) the impact of COVID-19 on cardiac diagnostic volumes in Latin America and (2) determine its relationship with COVID-19 case incidence and social distancing measures.

Methods: The International Atomic Energy Agency conducted a worldwide survey assessing changes in cardiac diagnostic volumes resulting from COVID-19. Cardiac diagnostic volumes were obtained from participating sites for March and April 2020 and compared to March 2019. Social distancing data were collected from Google COVID-19 community mobility reports and COVID-19 incidence per country from the Our World in Data.

Results: Surveys were conducted in 194 centers performing cardiac diagnostic procedures, in 19 countries in Latin America. Procedure volumes decreased 36\% from March 2019 to March 2020, and 82\% from March 2019 to April 2020. The greatest decreases occurred in echocardiogram stress tests (91\%), exercise treadmill tests (88\%), and computed tomography calcium scores (87\%), with slight variations between sub-regions of Latin America. Changes in social distancing patterns $(p<0.001)$ were more strongly associated with volume reduction than COVID-19 incidence $(p=0.003)$
\end{abstract}

Conclusions: COVID-19 was associated with a significant reduction in cardiac diagnostic procedures in Latin America, which was more related to social distancing than to the COVID-19 incidence. Better balance and timing of social distancing measures and planning to maintain access to medical care is warranted during a pandemic surge, especially in regions with high cardiovascular mortality.

Keywords: Cardiac Testing; Coronavírus; COVID-19; Cardiovascular Disease; Global Health.

Full texts in English - http://www.arquivosonline.com.br

\section{Resumo curto}

A pandemia de COVID-19 foi associada a uma redução significativa de procedimentos diagnósticos cardíacos na América Latina em abril de 2020, a qual foi mais relacionada às medidas distanciamento social do que ao aumento da incidência da COVID-19.
Pontos principais

- Em comparação com o mês de março de 2019, os volumes dos procedimentos diagnósticos cardíacos diminuíram 36\% em março de 2020 e 82\% em abril de 2020.

- As mudanças em padrões de distanciamento social $(p<0,001)$ estavam mais fortemente associadas com 
a redução volume do que a incidência de COVID-19 $(p=0,003)$.

- São necessários melhor equilíbrio e timing de medidas de distanciamento social e planejamento para manter o acesso ao atendimento médico durante um surto pandêmico, especialmente em regióes com alta mortalidade cardiovascular.

\section{Introdução}

As doenças cardiovasculares (DCV) continuam sendo a causa principal de mortalidade em todo o mundo, inclusive na América Latina. ${ }^{1,2}$ Embora as taxas de mortalidade tenham diminuído progressivamente nas últimas quatro décadas na maioria dos países de alta renda, o mesmo fenômeno não foi observado nos países de baixa e média renda, muitos dos quais se encontram na América Latina. ${ }^{3}$

Uma abordagem abrangente para lidar com as DCV e reduzir a mortalidade associada envolve prevenção adequada, incluindo controle de fatores de risco, uso apropriado de testes para diagnosticar e orientar o tratamento e o estabelecimento de terapias apropriadas. A Organização Mundial da Saúde recentemente chamou a atenção para a interrupção mundial da assistência à saúde causada pela pandemia de COVID-19, que infelizmente impõe uma carga adicional ao atendimento de pacientes com DCV em regiões como a América Latina que têm sido gravemente afetadas pela COVID-19. ${ }^{4}$

A International Atomic Energy Agency (IAEA) Division of Human Health visa apoiar os estados membros no combate às DCV, câncer, desnutrição e outras doenças por meio de prevenção, testes diagnósticos e tratamento adequados. Nesse sentido, a IAEA coordenou um levantamento mundial de centros de imagem cardiovascular (IAEA Noninvasive Cardiology Protocols Study of COVID-19, INCAPS COVID survey), com a finalidade de avaliar o impacto da pandemia na avaliação diagnóstica das DCV.

Os objetivos deste estudo foram: (1) avaliar o impacto da COVID-19 nos volumes de procedimentos diagnósticos cardíacos na América Latina e (2) determinar sua relação com a incidência de casos de COVID-19, apresentação temporal e intervenções de distanciamento social. Compreender a relação entre as fases da pandemia, as medidas de distanciamento social e o fornecimento de diagnóstico de DCV na América Latina é fundamental para melhor preparar para situações semelhantes no futuro.

\section{Métodos}

\section{Desenho do estudo}

Os dados deste estudo foram coletados como parte da pesquisa da IAEA sobre o impacto da COVID-19 em exames de imagem cardíaca (INCAPS COVID) e correlacionados com as métricas de distanciamento social que estão publicamente disponíveis nos Relatórios de mobilidade da comunidade de Google e a incidência mensal de COVID-19 a partir do banco de dados Our World in Data na América Latina. ${ }^{5-7}$ A pesquisa INCAPS COVID incluiu perguntas sobre a unidade de saúde, os profissionais de saúde, os equipamentos de proteção individual, os planos estratégicos para reabertura e as alterações nos volumes de procedimentos em relação a uma série de procedimentos diagnósticos cardiovasculares (Apêndice).

\section{Coleta de dados}

Com base na metodologia padronizada da IAEA, foi criado um sistema eletrônico de entrada de dados, empregando uma plataforma segura de software, o International Research Integration System (IRIS, https://iris. iaea.org). No estudo INCAPS COVID, não foram coletados dados confidenciais ou específicos dos pacientes e a participação dos locais de estudo foi voluntária; portanto, não foi considerada necessária a avaliação por comitê de ética externo.

Os participantes foram solicitados a fornecer estimativas dos volumes de procedimentos diagnósticos cardíacos para março de 2019, março de 2020 e abril de 2020, incluindo os seguintes: ecocardiografia transtorácica e transesofágica, ressonância magnética cardíaca (RMC), teste de estresse (teste ergométrico de esteira, teste de estresse ecocardiográfico, tomografia computadorizada por emissão de fóton único [SPECT], tomografia por emissão de pósitrons [PET] e RMC), estudos de infecção por PET, escore de cálcio por tomografia computadorizada, angiotomografia de artérias coronárias e angiografia coronária invasiva. Para fins de análise, dividimos a América Latina nas seguintes sub-regiões: América do Sul (Argentina, Bolívia, Brasil, Chile, Colômbia, Equador, Paraguai, Peru e Uruguai); América Central e México (Costa Rica, Guatemala, Honduras, México, Nicarágua, Panamá e El Salvador); e Caribe (Cuba, Jamaica e República Dominicana).

\section{Casos de COVID-19 por país}

Os números de casos de COVID-19 para cada país da América Latina foram baixados do site de acesso aberto Our World in Data (https://ourworldindata.org/coronavirussource-data), que coleta dados de diferentes fontes oficiais em todo o mundo. ${ }^{5}$ Our World in Data é um trabalho colaborativo entre pesquisadores da Universidade de Oxford baseado no Oxford Martin Programme on Global Development, que são os editores científicos do conteúdo do site, e a organização sem fins lucrativos Global Change Data Lab, que publica e mantém o site e as ferramentas de dados. Os dados coletados abrangem o período de fevereiro de 2020 a julho de 2020 para melhor refletir a evolução da pandemia na América Latina. Foi utilizado para análise o número de casos novos a cada mês por milhão de habitantes.

\section{Dados de mobilidade por país}

Os dados de mobilidade foram baixados dos Relatórios de mobilidade da comunidade de Google (https://www. google.com/covid19/mobility/) que agregam as tendências de mobilidade em 6 categorias diferentes: varejo e lazer, mercados e farmácias, parques, estações de transporte público, locais de trabalho e residencial. A linha de base foi o valor mediano, para o dia da semana correspondente, durante o período de 5 semanas entre o dia 3 de janeiro e o dia 6 de fevereiro de $2020 .{ }^{6}$ Google calcula essas informações com base nos dados de usuários que optaram pelo histórico de localização por meio de suas contas de Google; portanto, os dados representam uma amostra de todos os usuários. 
Utilizou-se a variação do tempo passado em casa (residencial) por mês como uma variável do "índice de imobilidade", de fevereiro de 2020 a julho de 2020, que reflete mudanças nos padrões de distanciamento social durante esse período. Os dados de mobilidade de Cuba não estavam disponíveis e, portanto, não foram utilizados nessas análises.

\section{Análise estatística}

As respostas às perguntas da pesquisa são apresentadas como números e porcentagens. O total de procedimentos por centro é apresentado como mediana e intervalo interquartil. A mudança percentual no volume de procedimentos foi comparada entre março de 2019 e março ou abril de 2020 utilizando o teste não paramétrico de Kruskal-Wallis com valores de $\mathrm{p}$ assintóticos e bilaterais. Para avaliar a associação das mudanças nos volumes de procedimentos cardíacos com as mudanças na mobilidade e a incidência da COVID-19, construímos uma equação de estimativa generalizada utilizando os países como unidades individuais e os números mensais de exames como o desfecho com o mês como variável de tempo. Foi realizada a análise estatística com Stata (versão 15.1, Stata Corporation, LLC, College Station, Texas), Microsoft Excel (2016) e foram construídos mapas coropléticos em R (versão 4.0.1, R Development Core Team, Viena, Áustria) utilizando os pacotes tmap e rnaturalearth.

\section{Resultados}

\section{Centros e redução de procedimentos}

Foram obtidos dados de 194 centros de internação e ambulatórios em 19 países da América Latina. Os maiores países regionais Brasil, Argentina e México também foram os que contribuíram com dados do maior número de centros: 70, 54 e 23 centros, respectivamente. As características de todos os centros estão resumidas na Tabela 1. Em total,

\section{Tabela 1 - Características dos centros da América Latina}

\begin{tabular}{|c|c|c|c|}
\hline & América do Sul & $\begin{array}{l}\text { América Central e } \\
\text { México }\end{array}$ & Caribe \\
\hline Países & 9 & 7 & 3 \\
\hline Número de centros & 155 & 31 & 8 \\
\hline Instituto de ensino (n, \%) & $69(44,5)$ & $21(67,7)$ & $4(50)$ \\
\hline Leitos hospitalares (mediana, IIQ) & $202,5(120-400)$ & $167(100-300)$ & $168(80-412,5)$ \\
\hline \multicolumn{4}{|l|}{ Tipo de instituto (n, \%) } \\
\hline Hospital, apenas internamento & $3(1,9)$ & $5(16,1)$ & $0(0)$ \\
\hline Hospital, apenas ambulatório & $3(1,9)$ & $0(0)$ & $0(0)$ \\
\hline Hospital, internação e ambulatório & $91(58,7)$ & $19(61,3)$ & $4(50)$ \\
\hline Centro de imagem ambulatorial & $45(29,0)$ & $2(6,4)$ & $2(25)$ \\
\hline Consultório médico ambulatorial & $13(8,4)$ & $5(16,1)$ & $2(25)$ \\
\hline \multicolumn{4}{|c|}{ Procedimentos totais por centro (mediana, IIQ) } \\
\hline Março 2019 & $157(67-502)$ & $91(38-430)$ & $173(53,5-559,5)$ \\
\hline Março 2020 & $89(31-253)$ & $35(19-143)$ & $147,5(24,5-343,5)$ \\
\hline Abril 2020 & $32(7-97)$ & $20(1-53)$ & $36(2,5-117)$ \\
\hline \multicolumn{4}{|c|}{ \% Redução de março de 2019 a abril de 2020} \\
\hline Ecocardiografia transtorácica & 80,1 & 54,0 & 87,0 \\
\hline Ecocardiografia transesofágica & 81,6 & 88,0 & 89,6 \\
\hline RMC & 77,2 & 80,9 & 100 \\
\hline Escore de cálcio por TC & 81,7 & 96,1 & 99,5 \\
\hline TC coronária & 73,0 & 84,8 & 85,9 \\
\hline Angiografia coronária invasiva & 63,8 & 77,7 & 70,8 \\
\hline Teste ergométrico de esteira & 88,4 & 84,9 & 95,6 \\
\hline Teste de estresse ecocardiográfico & 91,1 & 94,5 & 76,9 \\
\hline SPECT & 84,0 & 81,0 & 97,8 \\
\hline PET & 62,0 & 90,9 & NA \\
\hline RMC de estresse & 76,3 & 89,2 & NA \\
\hline
\end{tabular}

IIQ: intervalo interquartil; PET: tomografia por emissão de pósitrons; RMC: ressonância magnética cardiaca; SPECT: tomografia computadorizada por emissão de fóton único; TC: tomografia computadorizada. 
foram realizados 198.597 procedimentos diagnósticos cardíacos nos centros participantes durante os três meses considerados.

Na América Latina, em comparação com o mês de março de 2019, os volumes dos procedimentos diagnósticos cardíacos diminuíram 36\% em março de 2020 e 82\% em abril de 2020 (Figura 1 - Mapa). Houve alguma variação entre as regiões da América Latina e em relação ao tipo de procedimento cardíaco, com as maiores quedas no mês de abril de 2020, em relação aos testes de estresse ecocardiográfico (91\%), testes ergométricos de esteira (88\%) e escore de cálcio por tomografia computadorizada (87\%) (Tabela 1). Foram relatadas as menores reduções para angiografia coronária invasiva (67\%) e PET cardíaca (65\%). Os volumes de procedimento também diminuíram acentuadamente de março de 2020 a abril de 2020. Essas diminuições foram significativas $(p<0,001)$ em combinação (Figura 2) e para cada procedimento. Modelos lineares generalizados separados para as regiões da América Latina e em geral encontraram quedas significativas no volume de procedimentos ( $p<0,001$ ), usando modelos de regressão ponderados pelo volume de procedimentos de 2019. Nas 194 instalações de nosso estudo, estima-se que 129.030 procedimentos diagnósticos cardíacos, que teriam sido realizados com base nas taxas de procedimentos de março de 2019, não foram realizados durante esses dois meses da pandemia.

Dados de mobilidade, COVID-19 incidência e redução no volume de procedimentos

O maior aumento de tempo em casa (índice de imobilidade) ocorreu durante o mês de abril de 2020 na maioria dos países. Houve aumento médio de 26,7\% em abril que diminuiu para 18,8\% em julho, em comparação com a linha de base. As exceções foram Nicarágua e Chile, onde a imobilidade aumentou até junho (Figura 3).

No entanto, a pandemia de COVID-19 ainda estava em seus estágios iniciais na maioria dos países em abril de 2020, com um total de 199.277 casos até o final do mês. Na maioria dos países, os novos casos mensais de COVID-19 por milhão de habitantes continuaram aumentando e ainda não haviam atingido o pico em julho de 2020 (Figura 3). As exceções foram Cuba e Jamaica, que atingiram o pico em abril. Até o final de julho, o número de casos aumentou 23,5 vezes, totalizando 4.681 .377 casos confirmados de COVID-19. ${ }^{5}$

Tanto a redução da mobilidade quanto o aumento da incidência de COVID-19 estiveram associados à redução dos procedimentos diagnósticos cardíacos nos modelos generalizados ( $p<0,001)$. Ao ajustar um modelo

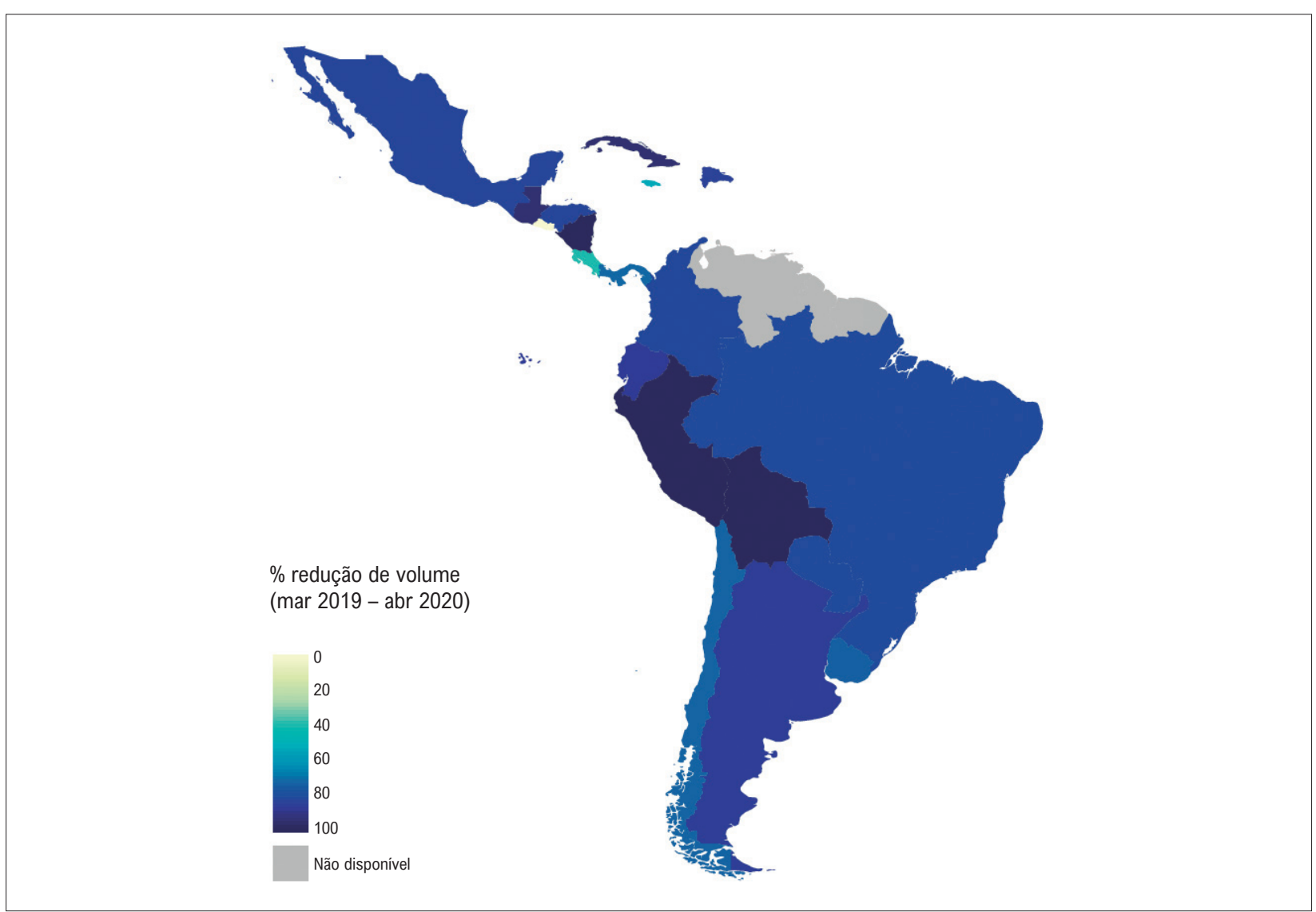

Figura 1 - Mapa com código de cores da América Latina mostrando a redução dos volumes totais de procedimentos diagnósticos cardiacos por país de março de 2019 a abril de 2020, no começo da pandemia de COVID-19. 


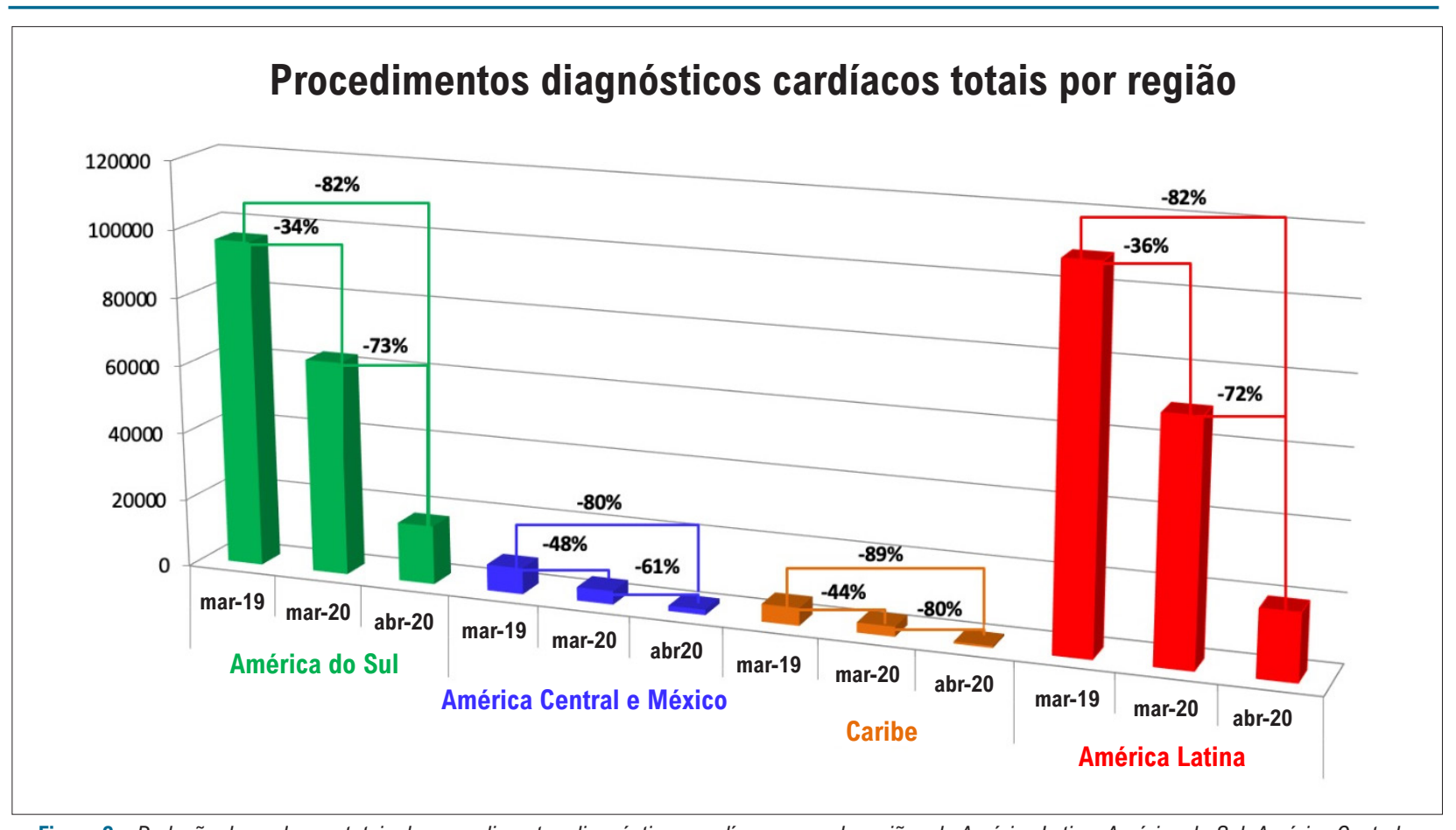

Figura 2 - Redução dos volumes totais de procedimentos diagnósticos cardiacos em sub-regiões da América Latina: América do Sul; América Central e México; e Caribe, em março de 2019, março de 2020 e abril de 2020, no começo da pandemia de COVID-19.

multivariável, a mobilidade ( $p<0,001$ ) foi mais fortemente associada à redução de volume do que a incidência de COVID-19 ( $p=0,003)$.

\section{Discussão}

Os resultados mostram uma redução significativa no número de procedimentos diagnósticos cardíacos realizados na América Latina durante a pandemia de COVID-19. A maior redução no número de procedimentos diagnósticos ocorreu no mês de menor mobilidade (abril), que por sua vez coincidiu com os períodos mais rígidos de quarentena de cada país.

Na maioria dos países da América Latina, o isolamento social foi introduzido em março, mesmo sem um número significativo de casos COVID-19. Em março e abril, houve uma queda maior no número de procedimentos diagnósticos cardíacos realizados na América Latina, em comparação com a Europa Ocidental (queda de 46\% em março e 69\% em abril) e os Estados Unidos e Canadá (queda de $39 \%$ em março e $68 \%$ em abril), apesar do fato de que estas regiões estavam experimentando o primeiro pico da pandemia, enquanto a América Latina estava apenas nos estágios iniciais. ${ }^{7}$ Até o final de março, haviam sido notificados menos de 250 casos em 9 países da região e, desde então, o número de casos continuou aumentando. Até o final de agosto de 2020, o SARS-CoV-2 havia comprometido todos os países da América Latina, com 7,15 milhões de pessoas afetadas. ${ }^{5} \mathrm{O}$ número real de casos pode ser ainda maior, já que o número de exames por milhão de pessoas continua baixo. ${ }^{8,9}$
Além da mortalidade direta causada pela COVID-19, houve preocupações crescentes em relação às consequências da pandemia de COVID-19 nos sistemas de saúde. ${ }^{10-12}$ O medo do contágio nos hospitais e centros de saúde pode ter levado à relutância dos pacientes em se submeter a procedimentos de diagnóstico cardíaco. Além disso, as intervenções e consultas eletivas tiveram que ser adiadas para priorizar as questões relacionadas à COVID-19 e evitar a exposição dos pacientes a um risco desnecessário de infecção em ambientes hospitalares ou ambulatoriais. ${ }^{13}$

A América Latina frequentemente enfrenta problemas de saúde que afetam principalmente os pobres, além de sistemas de saúde que já estão frágeis. ${ }^{14,15}$ Nessas condições, o distanciamento social retarda o pico da pandemia para permitir que os países com recursos de saúde limitados preparem-se para o diagnóstico e tratamento de pacientes em estado crítico. ${ }^{16,17}$ Apesar disso, de acordo com Walker e colegas, os países da América Latina que tiveram o primeiro pico da pandemia (Equador, México, Brasil, Chile, Bolívia, Panamá, Peru) ou com curvas de saúde não mitigadas/ limitadas (Peru, Chile, México, Equador) registraram uma taxa de mortalidade mais alta por milhão de habitantes. ${ }^{18}$ Isso poderia ser explicado pela incapacidade desses países de se prepararem para o pico da pandemia, com seus sistemas de saúde vulneráveis, resultando assim em altos índices de mortalidade. Eventos semelhantes ocorreram em países europeus atingidos pela primeira onda da pandemia de COVID-19, como a Itália e a Espanha.

No nível global, aproximadamente $70 \%$ das mortes por DCV ocorrem em países de baixa e média renda. ${ }^{3,19} \mathrm{O}$ grande intervalo de tempo entre o começo das medidas de 


\begin{tabular}{|c|c|c|c|c|c|c|c|c|c|c|c|c|c|}
\hline \multicolumn{7}{|c|}{ Número de casos novos de COVID-19 por mês por milhão de pessoas } & \multicolumn{7}{|c|}{ Índice de imobilidade por mês } \\
\hline & Fevereiro & Março & Abril & Maio & Junho & Julho & & Fevereiro & Março & Abril & Maio & Junho & Julho \\
\hline Brasil & 0 & 22 & 346 & 1.977 & 4.092 & 5.843 & Brasil & 1,67 & 8,00 & 17,27 & 15,65 & 13,93 & 12,43 \\
\hline Chile & 0 & 128 & 651 & 4,184 & 9.476 & 4,056 & Chile & 1,00 & 11,74 & 23,50 & 24,87 & 27,00 & 26,43 \\
\hline Panamá & 0 & 249 & 1.229 & 1.539 & 4.581 & 7.279 & Panamá & 1,27 & 17,39 & 39,67 & 35,52 & 29,67 & 29,86 \\
\hline Peru & 0 & 29 & 1.000 & 3.692 & 3.843 & 3.795 & Peru & 0,00 & 19,29 & 37,97 & 34,10 & 28,90 & 24,29 \\
\hline República Dominicana & 0 & 83 & 530 & 945 & 1.374 & 3.328 & República Dominicana & 1,40 & 12,90 & 29,17 & 22,71 & 15,70 & 12,86 \\
\hline Colômbia & 0 & 16 & 106 & 433 & 1.313 & 3.753 & Colômbia & $-0,60$ & 13,03 & 31,53 & 25,23 & 20,70 & 18,86 \\
\hline Bolívia & 0 & 9 & 86 & 727 & 1.930 & 3.693 & Bolívia & 1,93 & 16,00 & 37,70 & 33,45 & 27,60 & 27,00 \\
\hline Argentina & 0 & 21 & 73 & 264 & 1.019 & 2,583 & Argentina & 0,60 & 12,97 & 25,53 & 20,74 & 17,43 & 18,14 \\
\hline México & 0 & 9 & 130 & 541 & 1.033 & 1.517 & México & $-1,13$ & 5,61 & 19,77 & 19,42 & 16,20 & 14,43 \\
\hline Honduras & 0 & 14 & 64 & 437 & 1.386 & 2.283 & Honduras & $-0,67$ & 13,35 & 25,60 & 22,35 & 19,63 & 20,93 \\
\hline Equador & 0 & 111 & 1.287 & 788 & 969 & 1.627 & Equador & 2,13 & 18,77 & 34,93 & 29,16 & 22,27 & 20,29 \\
\hline Costa Rica & 0 & 65 & 75 & 66 & 436 & 2.752 & Costa Rica & $-1,27$ & 9,19 & 22,53 & 17,00 & 16,37 & 19,86 \\
\hline Guatemala & 0 & 2 & 31 & 232 & 707 & 1.754 & Guatemala & $-0,60$ & 12,61 & 25,57 & 24,74 & 22,60 & 23,64 \\
\hline El Salvador & 0 & 5 & 53 & 330 & 564 & 1.551 & El Salvador & $-0,53$ & 13,16 & 31,20 & 30,39 & 25,47 & 22,86 \\
\hline Paraguai & 0 & 9 & 26 & 100 & 172 & 423 & Paraguai & 0,20 & 13,65 & 26,60 & 18,26 & 13,67 & 12,43 \\
\hline Uruguai & 0 & 93 & 88 & 55 & 32 & 90 & Uruguai & 1,07 & 9,87 & 18,77 & 14,29 & 11,40 & 9,14 \\
\hline Jamaica & 0 & 13 & 121 & 63 & 40 & 56 & Jamaica & 1,93 & 9,39 & 22,77 & 19,35 & 14,20 & 12,43 \\
\hline Nicaragua & 0 & 0 & 0 & 5 & 6 & 6 & Nicaragua & $-0,53$ & 3,35 & 10,07 & 12,32 & 13,40 & 11,71 \\
\hline Cuba & 0 & 15 & 115 & 46 & 28 & 23 & Cuba & N/A & N/A & N/A & N/A & N/A & N/A \\
\hline Casos & & & & & & & Mobilidade & & & & & & \\
\hline $0 \square$ & & & & & & 10.000 & -1.27 & & & & & & 60,00 \\
\hline
\end{tabular}

Figura 3 - Painel direito: Número de casos novos de COVID-19 por mês por milhão de pessoas em 19 países da América Latina. Painel esquerdo: Mudança no tempo passado em casa ("índice de imobilidade") por mês, utilizando o período de 5 semanas do dia 3 de janeiro ao dia 6 de fevereiro de 2020 como linha de base em 18 países da América Latina (com exceção de Cuba). Nota-se que a maior imobilidade ocorreu em abril de 2020 na maioria dos países, em concordância com a queda abrupta dos procedimentos diagnósticos cardíacos. Por outro lado, o número de casos novos de COVID-19 por milhão de pessoas ainda estava aumentando progressivamente de março a julho de 2020 na maioria dos países.

distanciamento social e o primeiro pico da pandemia nos países da América Latina limitou o acesso dos pacientes aos procedimentos diagnósticos cardíacos, retardando ainda mais o diagnóstico e o tratamento oportuno das DCV. Na população da América Latina, essa cascata de eventos pode aumentar a morbimortalidade cardiovascular, conforme já tem sido relatado no Brasil..$^{20} \mathrm{~A}$ presente pesquisa não foi projetada para coletar informações sobre desfechos, mas o efeito negativo dos atrasos diagnósticos provavelmente será corroborado em estudos futuros para tais fins. Foi alcançada uma conclusão semelhante pela Sociedade LatinoAmericana de Cardiologia Intervencionista que realizou um estudo sobre a prática da Cardiologia Intervencionista durante a pandemia de COVID-19, com foco no infarto do miocárdio. ${ }^{21}$ Relataram uma redução de $51,2 \%$ no atendimento para infarto do miocárdio com elevação do segmento ST, com risco de aumento da morbimortalidade subsequente.

A infecção por COVID-19 pode estar associada a eventos cardiovasculares ou mimetizar doença cardíaca. ${ }^{22-27}$
Portanto, é essencial, durante a pandemia de COVID-19, manter a disponibilidade de todas as modalidades de diagnóstico cardíaco, em pacientes tanto positivos quanto negativos para COVID-19.

Existem várias lições que aprendemos para o futuro. Destacamos cinco: 1) Durante uma pandemia, deve ser mantido o acesso aos procedimentos diagnósticos cardíacos, tanto quanto possível, para toda a população, independentemente do tipo de restrição de mobilidade estabelecida em cada país, seguindo rigorosamente os devidos cuidados sanitários. 2) Campanhas educativas devem ser estabelecidas na mídia e nas redes sociais para explicar à comunidade a importância de buscar ajuda rapidamente diante dos sinais de alerta de doenças cardíacas, ao mesmo tempo em que se implementam medidas para prevenir a disseminação da COVID-19. 3) Áreas não COVID ("azul") para o atendimento de patologias não COVID e áreas para COVID ("vermelho") para pacientes infectados devem ser estabelecidas nos serviços de saúde. 4) É necessário garantir o acesso mundial aos suprimentos de saúde, de equipamentos 
de proteção individual a radiotraçadores. ${ }^{28}$ 5) Os governos devem garantir serviços de saúde não apenas aos pacientes com COVID, mas também aos pacientes sem COVID mas com DCV.

\section{Limitações}

A pesquisa INCAPS COVID-19 avaliou dados dos meses de março e abril de 2020, quando a pandemia ainda estava em fase inicial na maioria dos países da América Latina. Porém, de acordo com a evolução dos lockdowns, as datas de reabertura econômica e os dados de mobilidade de cada país, abril de 2020 foi o mês de menor atividade nesses países. A pesquisa foi realizada em um número limitado de hospitais e centros diagnósticos de cada país, com participação variável, o que poderia colocar em questão a representabilidade dos resultados. Apesar isso, a redução universal do número de procedimentos cardíacos não invasivos realizados em toda a América Latina sugere que a nossa amostra é representativa. Finalmente, dados de pesquisa de longo prazo para acompanhar toda a curva da pandemia não estavam disponíveis neste momento.

\section{Conclusão}

A COVID-19 foi associada a uma redução significativa e abrupta de procedimentos diagnósticos cardíacos na América Latina, a qual foi mais relacionada às medidas de distanciamento social do que ao aumento da incidência da doença. São necessários melhor equilíbrio e timing de medidas de distanciamento social e planejamento para manter o acesso a atendimento médico geral e atendimento cardiovascular em particular durante um surto pandêmico, especialmente em regiões com alta mortalidade cardiovascular.

\section{Agradecimentos}

O Grupo de Investigadores do INCAPS COVID, listado por nome no Apêndice, agradece às sociedades profissionais de cardiologia e imagem ao redor do mundo pelo seu apoio na divulgação da pesquisa para os seus membros. Estas incluem, mas não estão limitados às seguintes, em ordem alfabética: American Society of Nuclear Cardiology, Arab Society of Nuclear Medicine, Australasian Association of Nuclear Medicine Specialists, Australian and New Zealand Society of Nuclear Medicine, Belgian Society of Nuclear Medicine, British Society of Cardiovascular Imaging, Conjoint Committee for the Recognition of Training in CT Coronary Angiography, Consortium of Universities and Institutions in Japan, Danish Society of Cardiology, Gruppo Italiano di Cardiologia Nucleare, Indonesian Society of Nuclear Medicine, Japanese Society of Nuclear Cardiology, Moscow Regional Department of the Russian Nuclear Medicine Society, Philippine Society of Nuclear
Medicine, Russian Society of Radiology, Sociedad Española de Medicina Nuclear e Imagen Molecular, Sociedade Brasileira de Medicina Nuclear, Society of Cardiovascular Computed Tomography, e Thailand Society of Nuclear Medicine.

\section{Contribuição dos autores}

Concepção e desenho da pesquisa: Cerci RJ, Vitola JV, Paez D, Shaw LJ, Williams MC, Villines TC, Better N, Raggi P, Pascual TNB, Dondi M, Einstein AJ; Obtenção de dados: Cerci RJ, Vitola JV, Paez D, Zuluaga A, Bittencourt MS, Sierra-Galan LM, Carrascosa P, Campisi R, GutierrezVillamil C, Peix A, Chambers D, Velez MS, Alvarado CMG, Ventura ACF, Maldonado A, Castanos AP, Diaz TC, Herrera Y, Vasquez MC, Arrieta AA, Mut F, Goebel B, Cohen Y, Randazzo M, Shaw LJ, Williams MC, Villines TC, Better N, Dorbala S, Raggi P, Pascual TNB, Pynda Y, Dondi M, Einstein AJ, Malkovskiy E; Análise e interpretação dos dados: Cerci RJ, Paez D, Bittencourt MS, Sierra-Galan LM, Hirschfeld C, Cohen Y, Randazzo M, Shaw LJ, Williams MC, Villines TC, Better N, Dorbala S, Raggi P, Pascual TNB, Pynda Y, Dondi M, Einstein AJ; Análise estatística: Cerci RJ, Bittencourt MS, Hirschfeld C, Randazzo M, Pynda Y, Einstein AJ; Redação do manuscrito: Cerci RJ, Vitola JV, Zuluaga A, Sierra-Galan LM; Revisão crítica do manuscrito quanto ao conteúdo intelectual importante: Cerci RJ, Vitola JV, Paez D, Zuluaga A, Bittencourt MS, Sierra-Galan LM, Carrascosa P, Campisi R, GutierrezVillamil C, Peix A, Chambers D, Velez MS, Alvarado CMG, Ventura ACF, Maldonado A, Castanos AP, Diaz TC, Herrera Y, Vasquez MC, Arrieta AA, Mut F, Hirschfeld C, Goebel B, Cohen Y, Randazzo M, Shaw LJ, Williams MC, Villines TC, Better N, Dorbala S, Raggi P, Pascual TNB, Pynda Y, Dondi M, Einstein AJ, Malkovskiy E.

\section{Potencial conflito de interesse}

Não há conflito com o presente artigo

\section{Fontes de financiamento}

O presente estudo não teve fontes de financiamento externas.

\section{Vinculação acadêmica}

Não há vinculação deste estudo a programas de pósgraduação.

\section{Aprovação ética e consentimento informado}

Este artigo não contém estudos com humanos ou animais realizados por nenhum dos autores.

\section{Referências}

1. World Health Organization [Internet]. Cardiovascular Diseases. Geneva: World Health Organization; 2020. [cited 2021 Jul 20]. Available from: http:// www.who.int/cardiovascular_diseases/en/
2. Lanas F, Serón P, Lanas A. Coronary Heart Disease and Risk Factors in Latin America. Glob Heart. 2013;8(4):341-8. doi: 10.1016/j. gheart.2013.11.005. 
3. Benjamin EJ, Blaha MJ, Chiuve SE, Cushman M, Das SR, Deo R, et al. Heart Disease and Stroke Statistics-2017 Update: A Report From the American Heart Association. Circulation. 2017;135(10):146-603. doi: 10.1161/ CIR.0000000000000485.

4. World Health Organiation. [Internet]. World Health Organization Coronavirus Disease (COVID-19) Dashboard. Geneva: World Health Organization; 2020. [cited 2021 Jul 20]. Available from: https://covid19. who.int.

5. Coronavirus Pandemic (COVID-19). OurWorldinData. [Internet]. Oxford: OurWorldinData; 2020. [cited 2021 Jul 20]. Available from: https:// ourworldindata.org/coronavirus.

6. COVID-19 Community Mobility Reports. [Internet]. Menlo Park: Google; 2020. [cited 2021 Jul 20]. Available from: https://www.google.com/covid19/ mobility/data_documentation.html $\mathrm{hl}=\mathrm{en}$.

7. Einstein AJ, Shaw LJ, Hirschfeld C, Williams MC, Villines TC, Better $\mathrm{N}$, et al. International Impact of COVID-19 on the Diagnosis of Heart Disease. J Am Coll Cardiol. 2021;77(2):173-85. doi: 10.1016/j. jacc.2020.10.054

8. Oliveira TC, Abranches MV, Lana RM. Food (in)Security in Brazil in the Context of the SARS-CoV-2 Pandemic. Cad Saude Publica. 2020;36(4):e00055220. doi: 10.1590/0102-311X00055220.

9. Torres I, Sacoto F. Localising an Asset-Based COVID-19 Response in Ecuador. Lancet. 2020;395(10233):1339. doi: 10.1016/S01406736(20)30851-5.

10. Rosenbaum L. The Untold Toll - The Pandemic's Effects on Patients without Covid-19. N Engl J Med. 2020;382(24):2368-71. doi: 10.1056/ NEJMms2009984.

11. Baldi E, Sechi GM, Mare C, Canevari F, Brancaglione A, Primi R, et al. Out-of-Hospital Cardiac Arrest during the Covid-19 Outbreak in Italy. N Engl J Med. 2020;383(5):496-8. doi: 10.1056/NEJMc2010418.

12. Costa JA, Silveira JA, Santos SCMD, Nogueira PP. Cardiovascular Implications in Patients Infected with Covid-19 and the Importance of Social Isolation to Reduce Dissemination of the Disease. Arq Bras Cardiol. 2020;114(5):834-8. doi: 10.36660/abc.20200243.

13. Marijon E, Karam N, Jost D, Perrot D, Frattini B, Derkenne C, et al. Out-ofHospital Cardiac Arrest During the COVID-19 Pandemic in Paris, France: A Population-Based, Observational Study. Lancet Public Health. 2020;5(8):43743. doi: 10.1016/S2468-2667(20)30117-1.

14. Litewka SG, Heitman E. Latin American Healthcare Systems in Times of Pandemic. DevWorld Bioeth. 2020;20(2):69-73. doi: 10.1111/dewb.12262.

15. FAO: Hunger Increases in the World and in Latin America and the Caribbean for the Third Consecutive Year. [Internet]. Rome: Food and Agriculture Organization; 2018. [cited 2021 Jul 20]. Available from: http://www.fao.org/ americas/noticias/ver/en/c/1152157.

16. Benítez MA, Velasco C, Sequeira AR, Henríquez J, Menezes FM, Paolucci F. Responses to COVID-19 in Five Latin American Countries. Health Policy Technol. 2020;9(4):525-59. doi: 10.1016/j.hlpt.2020.08.014.
17. Díaz-Guio DA, Villamil-Gómez WE, Dajud L, Pérez-Díaz CE, BonillaAldana DK, Mondragon-Cardona A, et al. Will the Colombian Intensive Care Units Collapse Due to the COVID-19 Pandemic? Travel Med Infect Dis. 2020;38:101746. doi: 10.1016/j.tmaid.2020.101746.

18. Walker PGT, Whittaker C, Watson OJ, Baguelin M, Winskill P, Hamlet A, et al. The Impact of COVID-19 and Strategies for Mitigation and Suppression in Low- and Middle-Income Countries. Science. 2020;369(6502):413-22. doi: 10.1126/science.abc0035.

19. Roth GA, Huffman MD, Moran AE, Feigin V, Mensah GA, Naghavi M, et al. Global and Regional Patterns in Cardiovascular Mortality from 1990 to 2013. Circulation. 2015;132(17):1667-78. doi: 10.1161/ CIRCULATIONAHA.114.008720.

20. Brant LCC, Nascimento BR, Teixeira RA, Lopes MACQ, Malta DC, Oliveira GMM, et al. Excess of Cardiovascular Deaths During the COVID-19 Pandemic in Brazilian Capital Cities. Heart. 2020;106(24):1898-905. doi: 10.1136/heartjnl-2020-317663.

21. Mayol J, Artucio C, Batista I, Puentes A, Villegas J, Quizpe R, et al. An International Survey in Latin America on the Practice of Interventional Cardiology During the COVID-19 Pandemic, with a Particular Focus on Myocardial Infarction. Neth Heart J. 2020;28(7-8):424-30. doi: 10.1007/ s12471-020-01440-y.

22. Nishiga M, Wang DW, Han Y, Lewis DB, Wu JC. COVID-19 and Cardiovascular Disease: From Basic Mechanisms to Clinical Perspectives. Nat Rev Cardiol. 2020;17(9):543-58. doi: 10.1038/s41569-020-0413-9.

23. Shi S, Qin M, Shen B, Cai Y, Liu T, Yang F, et al. Association of Cardiac Injury with Mortality in Hospitalized Patients with COVID-19 in Wuhan, China. JAMA Cardiol. 2020;5(7):802-10. doi: 10.1001/ jamacardio.2020.0950.

24. Guo T, Fan Y, Chen M, Wu X, Zhang L, He T, et al. Cardiovascular Implications of Fatal Outcomes of Patients With Coronavirus Disease 2019 (COVID-19). JAMA Cardiol. 2020;5(7):811-8. doi: 10.1001/ jamacardio.2020.1017.

25. Shi S, Qin M, Cai Y, Liu T, Shen B, Yang F, et al. Characteristics and Clinical Significance of Myocardial Injury in Patients with Severe Coronavirus Disease 2019. Eur Heart J. 2020;41(22):2070-9. doi: 10.1093/eurheartj/ ehaa408.

26. ESC Guidance for the Diagnosis and Management of CV Disease during the COVID-19 Pandemic. [Internet]. Bruxelas: Europe Society of Cardiology; 2020. [cited 2021 Jul 20]. Available from: https://www. escardio.org/Education/COVID-19-and-Cardiology/ESC-COVID-19Guidance.

27. Costa IBSDS, Bittar CS, Rizk SI, Araújo Filho AE, Santos KAQ, Machado TIV, et al. The Heart and COVID-19: What Cardiologists Need to Know. Arq Bras Cardiol. 2020;114(5):805-16. doi: 10.36660/abc.20200279.

28. Freudenberg LS, Paez D, Giammarile F, Cerci J, Modiselle M, Pascual TNB, et al. Global Impact of COVID-19 on Nuclear Medicine Departments: An International Survey in April 2020. J Nucl Med. 2020;61(9):1278-83. doi: 10.2967/jnumed.120.249821.

\section{* Material suplementar}

Para informação adicional, por favor, clique aqui. 